\title{
Adaptive Control of the Boost Inverter with Load RL *
}

\author{
Carolina Albea* Francisco Gordillo* \\ Carlos Canudas-de-Wit ${ }^{* *}$ \\ * Universidad de Sevilla, Dpto. Ingeniería de Sistemas y Automática, \\ Camino de los Descubrimientos, s/n, 41092 Sevilla, Spain \\ (e-mail: calbea@cartuja.us.es, gordillo@esi.us.es) \\ ** CNRS, Gipsa-lab Grenoble. ENSIEG-BP 46, 38402 Saint Martin \\ d'Hères Cedex, France, \\ (e-mail:carlos.canudas-de-wit@resulting inpg.fr)
}

\begin{abstract}
This paper proposes an adaptive control for the nonlinear boost inverter with nonresistive load in order to cope with unknown resistive load as well as unknown inductive load. The adaptive control is accomplished by using a state observer to one side of the inverter with a quadratic Lyapunov function and by measuring the states variables. The stability of the complete system is analyzed by means of singular perturbation analysis. The adaptation of the both parameters is tested by using simulations.
\end{abstract}

\section{INTRODUCTION}

The control of switched-mode power converters (SMPC) with AC output is usually accomplished tracking a reference (sinusoidal) signal Sanchis et al. [2005], Vazquez et al. [2003]. The use of this external signal makes from the mathematical point of view the closed-loop control system to be non-autonomous and thus, making its analysis involved. A different approach was used in Pagano et al. [2005]: a control law was designed for stabilizing a limit cycle corresponding to the desired behavior. No external signals were needed. In Albea et al. [2006] this idea was applied to the boost inverter Caceres and Barbi [1999]. A Phase-Lock Loop (PLL) is added to the control law in order to achieve synchronization between both parts of the circuit as well as synchronize the voltage output with a pre-specified signal, e.g. synchronization with the electrical grid. Only the case of known resistive load was considered.

The unknown load problem in SMPC is usually dealt with adaptation mechanisms combined with other techniques such as discontinuous feedback regulators, backstepping with passivity-based controllers, sliding mode, El Fadil et al. [2003], Tan et al. [2005]. In Pagano et al. [2005] is computed an adaptive control for the case of the boost converter. The controller used in such paper follows the idea given above. The fact that the boost inverter model is 4-dimensional and nonlinear makes the design of the adaptation law more involved. A state observer for some of the converter variables is designed even when the state variables are measured. In order to analyze the stability of the full system singular perturbation analysis is used Khalil [2002]. For simplicity, the PLL is not considered in this analysis.

The case of non-resistive load was studied in Albea and Gordillo [2007], but only for known load. In this paper,

\footnotetext{
‡ This work was supported by the MEC-FEDER grant DPI200607338 .
}

the previous results are extended to case of unknown and non-resistive load using the adaptation mechanism from Albea et al. [2007], adapting the parameters of the load at the same time. Singular perturbation analysis was used to prove the stability of the full system. The resultant adaptive control is tested by means of simulations.

The rest of the paper is organized as follows: in Sect. 2 the model of the double boost converter (boost inverter) is presented. Section 3 states the problem, which is solved in Sect. 4 by means of the design of the adaptation mechanism. Section 5 is devoted to the stability analysis and Sect. 6 presents some simulation results. The paper closes with a section of conclusions.

\section{BOOST INVERTER MODEL}

The boost inverter Caceres and Barbi [1999] is specially interesting because it can generates an ac output voltage larger than the its dc input Caceres and Barbi [1999]. It is composed of two dc-dc converters and a load connected across of them. Each converter produces a dc-biased sine wave output, $v_{1}$ and $v_{2}$, so that each source generates an unipolar voltage. Voltages $v_{1}$ and $v_{2}$ should present a phase shift equal to $180^{\circ}$, which maximizes the voltage excursion across the load. The circuit implementation is shown in Fig. 1. By this mean it is possible to generate an oscillatory signal without bias. In Albea et al. [2006] the load was resistive, and in Albea and Gordillo [2007] was studied this same circuit with a RL load.

Here it is assumed that:

- all the components are ideal and the currents of the converter are continuous,

- the inductances $L=L_{1}=L_{2}$, and the capacitances $C=C_{1}=C_{2}$, are known and symmetric,

- the load $R_{0}$ and $L_{R 0}$ are unknown, and it has to be estimated. 


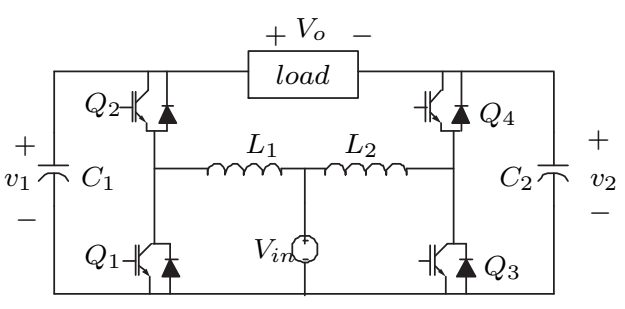

Fig. 1. Boost inverter model

The boost dc-ac converter can be simplified as shown in Fig. 2. This simplification lets see clearer the bidirectional

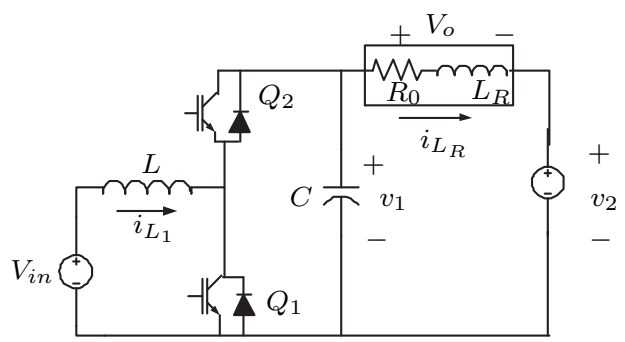

Fig. 2. Simplified Boost inverter model

current of each boost dc-dc converter.

The circuit in Fig. 1 is driven by the transistor ON/OFF inputs $Q_{i}$. This yields two modes of operation illustrated in Appendix A. Formally this yields a switched model which is more involved. For control purposes, it is common to use an averaged model described in terms of the mean currents and voltages values. This model is more suited for control because it is described by a "continuous" time smooth and nonlinear ODE. Following Albea et al. [2006], this averaging process yields the normalized model described below.

\subsection{Normalized averaged model}

In order to simplify the study, we can yield a normalized model by using the change of variables:

$$
\begin{aligned}
& x_{1}=\frac{1}{V_{i n}} \sqrt{\frac{L}{C}} i_{L_{1}} \\
& x_{2}=\frac{v_{1}}{V_{\text {in }}} \\
& x_{5}=\frac{1}{V_{\text {in }}} \sqrt{\frac{\hat{L}_{R}}{C}} i_{L_{1}}
\end{aligned}
$$

and defining the new time variable with $\tilde{t}=\frac{1}{\sqrt{L C}} t$.

Assuming an unknown RL load with a averaged current $x_{5}$, a normalized model in terms of the averaged current $x_{1}$ and the averaged voltage $x_{2}$, for one side of the inverter (see, Albea and Gordillo [2007]), is:

$$
\begin{aligned}
& \dot{x}_{1}=-u_{1} x_{2}+1 \\
& \dot{x}_{2}=u_{1} x_{1}-b x_{5} \\
& \dot{x}_{5}=-\theta_{1} x_{5}+\theta_{2} c x_{2}-\theta_{2} c x_{4}+a \dot{\hat{\theta}}_{2} x_{5}
\end{aligned}
$$

where $\theta_{1}=\frac{R \sqrt{L C}}{L_{R}}, \theta_{2}=\frac{1}{L_{R}}, \hat{\theta}_{2}=\frac{1}{\hat{L}_{R}}, a=\frac{1}{2} \hat{L}_{R}^{2} \operatorname{Vin} \sqrt{C}$, $b=\sqrt{\frac{L}{\hat{L}_{R}}}$ and $c=\sqrt{L \hat{L}_{R}}$ and the respective normalized model for the other side is obtained similarly by symmetry

$$
\begin{aligned}
& \dot{x}_{3}=-u_{2} x_{4}+1 \\
& \dot{x}_{4}=u_{2} x_{3}-b x_{5}
\end{aligned}
$$

where $u_{i} \in[0,1], i=1,2$ describes the control inputs. Note that they are also normalized and reflect the mean duty-cycle activation percent of each circuit. They are here treated as "continuous" variables. Parameters $a, b$ and $c$ depends on the estimation of $L_{R}$ and is assumed to be known. $\theta_{1}$ and $\theta_{2}$ depends on the unknown load which is unknown.

\section{PROBLEM FORMULATION}

The control problem is to design a control law for $u_{1}$, and $u_{2}$, for the system (1)-(3) and (4)-(5) in order to make the output $y$ to oscillate as a sinusoidal signal with a given amplitude i.e.

$$
y=x_{2}-x_{4} \quad \rightarrow \quad y_{r}=A \cos (\omega t+\varphi)
$$

with a pre-specified value for $A$ and $\omega$. The phase shift $\varphi$ is not specified.

Under the assumption that $a, b, c, \theta_{1}$ and $\theta_{2}$ are constants and knowns, in Albea and Gordillo [2007] a nonlinear control law based on Hamiltonian approach was proposed. The design is based on the following change of coordinates:

$$
\begin{aligned}
\eta_{1} & =\frac{x_{1}^{2}+x_{2}^{2}}{2} \\
\eta_{2} & =x_{1}-b x_{2} x_{5}+\eta_{20} \\
\eta_{3} & =\frac{x_{3}^{2}+x_{4}^{2}}{2} \\
\eta_{4} & =x_{3}+b x_{4} x_{5}+\eta_{40}
\end{aligned}
$$

The controller recalled further below, has as an objective to render the following functions tend to zero

$$
\begin{aligned}
& \Gamma_{1} \equiv \omega^{2}\left(\eta_{1}-\eta_{10}\right)^{2}+\left(\eta_{2}-\eta_{20}^{2}\right)^{2}-\mu=0 \\
& \Gamma_{2} \equiv \omega^{2}\left(\eta_{3}-\eta_{30}\right)^{2}+\left(\eta_{4}-\eta_{40}^{2}\right)^{2}-\mu=0
\end{aligned}
$$

orbitally stable. The parameters $\eta_{10}, \eta_{20}$ and $\eta_{30}, \eta_{40}$ define the respective ellipse centers and $\omega, \mu$ are related to their size. Based on this definition, the nonlinear control law as proposed in Albea and Gordillo [2007] has the following form:

$$
u=k\left(x, \theta_{1}, \theta_{2}\right)=\left[\begin{array}{l}
u_{1} \\
u_{2}
\end{array}\right]=\left[\begin{array}{l}
k_{1}\left(x, \theta_{1}, \theta_{2}\right) \\
k_{2}\left(x, \theta_{1}, \theta_{2}\right)
\end{array}\right] \quad u \in \mathbb{R}^{2}
$$

with $k_{1}\left(x, \theta_{1}, \theta_{2}\right), k_{2}\left(x, \theta_{1}, \theta_{2}\right)$ given in Appendix B.

The design is completed with an additional outer loop (PLL) that has the function of achieving a phase shift equal to $180^{\circ}$ between the two voltages $v_{1}$, and $v_{2}$ reaching in that way the desired objective. The goal here is to extent this work to the case of unknown load.

\section{ADAPTATION LAW LOAD DESIGN}

In this section we propose an adaptive law (or a load observer) to cope with load variations and/or uncertainties 
on the load parameters $\theta_{1}$, and $\theta_{2}$. This observer is designed based only on one-side of the circuit, which contains enough information to make this parameter observable. Therefore the use of the full two-side circuit is not necessary at this stage.

The one-side (left) circuit (1)-(3), can be rewritten compactly as:

$$
\begin{aligned}
\dot{x}_{l} & =U_{l} x_{l}+b B_{l} x_{5}+c \theta_{2} C_{l} y+\left(a \hat{\theta}_{2}-\theta_{1}\right) C_{l} x_{5}+E_{l}(12 \\
y & =x_{2}-x_{4}
\end{aligned}
$$

with $x_{l}=\left[x_{1}, x_{2}, x_{5}\right]^{T}$, and

$$
U_{l}=\left[\begin{array}{ccc}
0 & -u_{1} & 0 \\
u_{1} & 0 & 0 \\
0 & 0 & 0
\end{array}\right], B_{l}=\left[\begin{array}{c}
0 \\
-1 \\
0
\end{array}\right], E_{l}=\left[\begin{array}{l}
1 \\
0 \\
0
\end{array}\right], C_{l}=\left[\begin{array}{l}
0 \\
0 \\
1
\end{array}\right]
$$

where $\theta_{1}$ and $\theta_{2}$ are disturbed parameters.

In what follows, we assume that both voltages and currents are measurable (either analogically or numerically), and thus accessible for control use.

\subsection{Adaptation law}

The proposed adaptation law is composed by: state observers, plus adaptation laws for $\theta_{1}$ and $\theta_{2}$. It has the following structure:

$$
\begin{aligned}
\dot{\hat{x}}= & U_{l} \hat{x}+b B_{l} x_{5}+c \hat{\theta}_{2} C_{l} y+\left(a \hat{\theta}_{2}-\hat{\theta}_{1}\right) C_{l} x_{5}+ \\
& E_{l}+K\left(x_{l}-\hat{x}\right) \\
\dot{\hat{\theta}}_{1}= & \beta_{1}\left(x_{l}, \hat{x}\right) \\
\dot{\hat{\theta}}_{2}= & \beta_{2}\left(x_{l}, \hat{x}\right)
\end{aligned}
$$

where $K \in \mathbb{R}^{3 \times 3}$ is a constant design matrix, and $\theta_{1}\left(x_{l}, \hat{x}\right)$ and $\theta_{2}\left(x_{l}, \hat{x}\right)$ are the adaptation laws to be designed. Note, that even if $x$ is accessible, the adaptation law designed here requires the additional (or extended) state observer. This will become clear during the analysis of the error equation system, as studied below.

\subsection{Error equation}

Assume that $\theta_{1}$ and $\theta_{2}$ are constant parameters $\left(\dot{\theta}_{1}=0\right.$ and $\dot{\theta}_{2}=0$ ) (or that change slowly $\dot{\theta}_{1} \approx 0$ and $\dot{\theta}_{2} \approx 0$ ) and define the following error variables:

$$
\tilde{x}=x_{l}-\hat{x}, \quad \tilde{\theta}_{1}=\theta_{1}-\hat{\theta}_{1}, \quad \dot{\tilde{\theta}}_{1}=-\dot{\hat{\theta}}_{1} \quad \tilde{\theta}_{2}=\theta_{2}-\hat{\theta}_{2}, \quad \dot{\tilde{\theta}}_{2}=-\dot{\dot{\theta}_{2}}
$$

Error equation are now derived from (12)-(13) together with (15)-(17)

$$
\begin{aligned}
\dot{\tilde{x}} & =-K \tilde{x}+c \tilde{\theta}_{2} C_{l} y-\tilde{\theta}_{1} C_{l} x_{5} \\
\dot{\tilde{\theta}}_{1} & =-\beta_{1}\left(x_{l}, \hat{x}\right) \\
\dot{\tilde{\theta}}_{2} & =-\beta_{2}\left(x_{l}, \hat{x}\right)
\end{aligned}
$$

Let $K$ be of the form,

$$
K=\alpha I, \quad \alpha>0
$$

and $P=I$ be the trivial solution of $P K^{T}+K P=-Q$, with $Q=-2 \alpha I$.
Now introducing

$$
V=\tilde{x}^{T} P \tilde{x}+\frac{\tilde{\theta}_{1}^{2}}{\gamma_{1}}+\frac{\tilde{\theta}_{2}^{2}}{\gamma_{2}}
$$

it follows that

$$
\begin{aligned}
\dot{V} & =\tilde{x}^{T} Q \tilde{x}+2 \tilde{\theta}_{1}\left(-\tilde{x}^{T} P C_{l} x_{5}+\frac{\dot{\tilde{\theta}}_{1}}{\gamma_{1}}\right)+2 \tilde{\theta}_{2}\left(\tilde{x}^{T} P c C_{l} y+\frac{\dot{\tilde{\theta}}_{2}}{\gamma_{2}}\right) \\
& =\tilde{x}^{T} Q \tilde{x}+2 \tilde{\theta}_{1}\left(-\tilde{x}^{T} P C_{l} x_{5}-\frac{\dot{\tilde{\theta}}_{1}}{\gamma_{1}}\right)+2 \tilde{\theta}_{2}\left(\tilde{x}^{T} P c C_{l} y-\frac{\dot{\tilde{\theta}}_{2}}{\gamma_{2}}\right)
\end{aligned}
$$

The adaptation laws are now designed by canceling the terms in square brackets, i.e.

$$
\begin{aligned}
& \dot{\hat{\theta}}_{1}=\gamma_{1}\left(-\tilde{x}^{T} P C_{l} x_{5}\right) \\
& \dot{\hat{\theta}}_{2}=\gamma_{2} \tilde{x}^{T} P c C_{l} y
\end{aligned}
$$

The stability properties of the observer and the adaptive law error equations were discussed in Albea et al. [2007] reaching to:

Lemma 1. Consider the open-loop system (12)-(13), and assume that its solutions are bounded. The extended observer (15)-(17) has the following properties:

i) The estimated states $\hat{x}, \hat{\theta}_{1}, \hat{\theta}_{2}$ are bounded.

ii) $\lim _{t \rightarrow \infty} \hat{x}(t)=x(t)$.

iii) $\lim _{t \rightarrow \infty} \hat{\theta}_{1}(t)=\theta_{1} \Longleftrightarrow y(t) \not \equiv 0, x_{5}(t) \not \equiv 0 \forall t \geq 0$.

iv) $\lim _{t \rightarrow \infty} \hat{\theta}_{2}(t)=\theta_{2} \Longleftrightarrow y(t) \not \equiv 0, x_{5}(t) \not \equiv 0 \forall t \geq 0$.

\section{STABILITY OF THE FULL CLOSED-LOOP EQUATIONS}

The stability of the complete system (the system state variables plus the observer extended) is analyzed in this section.

The open-loop two-sides inverter (1)-(3) and (4)-(5), can be compactly rewritten as:

$$
\begin{aligned}
& \dot{x}=U x+b B x_{5}+c \theta_{2} C y+\left(a \hat{\theta}_{2}-\theta_{1}\right) C x_{5}+E \\
& y=x_{2}-x_{4}
\end{aligned}
$$

with $x=\left[x_{1}, x_{2}, x_{3}, x_{4}, x_{5}\right]^{T}$, and

$$
U=\left[\begin{array}{ccccc}
0 & -u_{1} & 0 & 0 & 0 \\
u_{1} & 0 & 0 & 0 & 0 \\
0 & 0 & 0 & -u_{2} & 0 \\
0 & 0 & u_{2} & 0 & 0 \\
0 & 0 & 0 & 0 & 0
\end{array}\right], B=\left[\begin{array}{c}
0 \\
-1 \\
0 \\
1 \\
0
\end{array}\right], C=\left[\begin{array}{l}
0 \\
0 \\
0 \\
0 \\
1
\end{array}\right], E=\left[\begin{array}{l}
1 \\
0 \\
1 \\
0 \\
0
\end{array}\right]
$$

\subsection{Tuned System}

The tuned system is defined as the ideal closed-loop system under the action of the tuned feedback law $u^{*}=$ $k\left(x, \theta_{1}, \theta_{2}\right)$, computed with the exact value of $x, \theta_{1}$ and $\theta_{2}$.

The tuned systems given in Albea and Gordillo [2007] writes

$$
\begin{aligned}
\dot{x} & =U\left(u^{*}\right) x+b B x_{5}+c \theta_{2} C y+\left(a \hat{\theta}_{2}-\theta_{1}\right) C x_{5}+E \\
& =U\left(k\left(x, \theta_{1}, \theta_{2}\right)\right) x+b B x_{5}+c \theta_{2} C y+\left(a \hat{\theta}_{2}-\theta_{1}\right) C x_{5}+E \\
& =f(x)
\end{aligned}
$$


and it achieves an asymptotically orbitally stable periodic solutions, i.e.

$$
x^{*}(t)=x^{*}(t+T)
$$

In Albea et al. [2006] it has been shown that the functions $\Gamma_{1}$ and $\Gamma_{2}$ defined in (10)-(11) tend to zero. They correspond to periodic sinusoidal solutions with period $T=2 \pi / \omega$. Consequently, $y^{*}=x_{2}^{*}-x_{4}^{*}$ is also sinusoidal.

\subsection{Closed-loop system}

How it is seen in Albea et al. [2007], note that control law depends on the estimation as $\hat{u}=k\left(x, \hat{\theta}_{1}, \hat{\theta}_{2}\right)$. Note that this control law depends on the state $x$ and not on its estimation $\hat{x}$, because the state $x$ is directly measured. The role of $\hat{x}$ is then just to make possible the design of the adaptation law for $\theta_{1}$ and $\theta_{2}$.

The closed-loop equation resulting from the use of $\hat{u}=$ $k\left(x, \hat{\theta}_{1}, \hat{\theta}_{2}\right)$ writes, as

$$
\begin{aligned}
\dot{x} & =U(\hat{u}) x+b B x_{5}+c \theta_{2} C y+\left(a \hat{\theta}_{2}-\theta_{1}\right) C x_{5}+E \pm U\left(u^{*}\right) x \\
& =f(x)+\left[U(\hat{u})-U\left(u^{*}\right)\right] x \\
& =f(x)-U(\tilde{u}) x
\end{aligned}
$$

where $\tilde{u}=u^{*}-\hat{u}$. Note that $U(\tilde{u})=U\left(\tilde{x}, \tilde{\theta}_{1}, \tilde{\theta}_{2}\right)$, captures the mismatch between the estimated and the true value of the load (see Albea et al. [2007]). This term has the following property:

Property 1. Let $\mathbb{M}=\left\{\left(x, \tilde{\theta}_{1}, \tilde{\theta}_{2}\right):|| x-x^{*} \|<\epsilon_{x},\left|\tilde{\theta}_{1}\right|<\right.$ $\left.\epsilon_{\theta_{1}},\left|\tilde{\theta}_{2}\right|<\epsilon_{\theta_{2}}\right\}$, be a compact domain including the asymptotic periodic solutions of the tuned system and the exact load. Then, the function $U(\tilde{u})=U\left(x, \tilde{\theta}_{1}, \tilde{\theta}_{2}\right)$ has $\forall\left(x, \tilde{\theta}_{1}, \tilde{\theta}_{2}\right) \in \mathbb{M}$, the following properties:

i) it is continuous, analytic, and free of singularities

ii) it has the following limits:

$$
\lim _{\tilde{u} \rightarrow 0} U(\tilde{u})=\lim _{\substack{\tilde{\theta}_{1} \rightarrow 0 \\ \tilde{\theta}_{2} \rightarrow 0 \\ \tilde{x} \rightarrow x}} U\left(x, \tilde{\theta}_{1}, \tilde{\theta}_{2}\right)=0
$$

Putting together the closed-loop equation resulting from (25) with the observer error system give the complete set, with $y=y(x)$

$$
\begin{aligned}
\dot{x} & =f(x)-U\left(x, \tilde{\theta}_{1}, \tilde{\theta}_{2}\right) x \\
\dot{\tilde{x}} & =-\alpha \tilde{x}+c\left(\hat{\theta}_{2}\right) \tilde{\theta}_{2} C_{l} y-\tilde{\theta}_{1} C_{l} x_{5} \\
\dot{\tilde{\theta}} & =\gamma_{1}\left(-\tilde{x}^{T} P C_{l} x_{5}\right) \\
\dot{\tilde{\theta}}_{2} & =\gamma_{2} \tilde{x}^{T} P c\left(\hat{\theta}_{2}\right) C_{l} y
\end{aligned}
$$

we have substituted $K=\alpha I$. The stability consideration discussed here will be based on the time-scale separation. The main idea is that with the suited choice of gains (as discussed latter) the observer equation (28)-(30) can be seen as the fast variables and the equation (27) as the slow subsystem. Note again, that this time-scale separation should be enforced by a particular choice of the observer and adaption gains: $\alpha, \gamma_{2}$ and $\gamma_{1}$.

\subsection{Singular perturbed form}

To put the system above in the standard singular perturbation form, we follow the next steps:
- introduce $\bar{\theta}_{1}=\frac{\tilde{\theta}_{1}}{\alpha}, \bar{\theta}_{2}=\frac{\tilde{\theta}_{2}}{\alpha}$

- select $\gamma=\gamma_{1}=\gamma_{2}=\alpha^{2}$

- define $\varepsilon=\frac{1}{\alpha}$

With these considerations, we achieve,

$$
\begin{aligned}
\dot{x} & =f(x)-U(x, \bar{b}, \bar{c}) x \\
\varepsilon \dot{\tilde{x}} & =-\tilde{x}+c\left(\bar{\theta}_{2}\right) \bar{\theta}_{2} C_{l} y-\bar{\theta}_{1} C_{l} x_{5} \\
\varepsilon \dot{\bar{\theta}}_{1} & =-\tilde{x}^{T} P C_{l} x_{5} \\
\varepsilon \dot{\bar{\theta}}_{2} & =\tilde{x}^{T} P c\left(\bar{\theta}_{2}\right) C_{l} y
\end{aligned}
$$

where $\varepsilon>0$ being the small parameters. Note that this particular selection of gains imposes relationships for the adaptation $\gamma$. The target system for the slow variables, defined after the change of coordinates (6)-(9) Albea et al. [2006], is

$$
\begin{aligned}
& \dot{\eta}_{1}=\omega \eta_{2} \\
& \dot{\eta}_{2}=-\omega \eta_{1}-k \eta_{2} \Gamma\left(\eta_{1}, \eta_{2}\right) .
\end{aligned}
$$

Dividing this equations by $\omega^{2}$ they achieve a similar form to fast variables equations. As we want that variable $x$ is much slower than $\tilde{x}, \bar{\theta}_{1}$ and $\bar{\theta}_{2}$, we have to impose

$$
\varepsilon \ll \frac{1}{\omega}, \quad \varepsilon \ll \frac{1}{k} .
$$

This means that the adaptation gain $\gamma$ as well as the tuning parameter $k$ should be related to the desired frequency as:

$$
\gamma \gg \omega^{2}, \quad \gamma \gg k^{2}
$$

Letting $z=\left[\tilde{x}, \bar{\theta}_{1}, \bar{\theta}_{2}\right]^{T}$ gives the general form

$$
\begin{aligned}
\dot{x} & =f(x)-U(\tilde{u}) x \\
\varepsilon \dot{z} & =g(x, z)
\end{aligned}
$$

with, $x\left(t_{0}\right)={ }_{1} x^{0}, x \in \mathbb{R}^{5}, z\left(t_{0}\right)=z^{0}, z \in \mathbb{R}^{5}$, and $g(z, x)=\varepsilon\left[\tilde{x}, \bar{\theta}_{1}, \bar{\theta}_{2}\right]^{T}$ According to the singular perturbation analysis, we need to follow the next steps:

(1) Find a stationary solution of the fast subsystem (32) by finding roots of the equation $g(x, z)=0$, i.e. $z=\phi(x)$

(2) Substitute this solution in the slow subsystem (31), and find a the resulting slow system

$$
\dot{x}=f(x)-U(\tilde{u}(x, \phi(x))) x
$$

(3) Check the boundary layer properties of the fast subsystem along one particular solution of $\dot{x}=f(x)-$ $U(\tilde{u}(x, \phi(x))) x$.

\subsection{Slow sub-system}

Proceeding to the steps 1 and 2 above requires to find the roots of $g(x, z)=0$, which are calculated from

$$
\begin{aligned}
\tilde{x} & =c\left(\bar{\theta}_{2}\right) \bar{\theta}_{2} C_{l} y-\bar{\theta}_{1} C_{l} x_{5} \\
0 & =-\left(c\left(\bar{\theta}_{2}\right) \bar{\theta}_{2} C_{l} y-\bar{\theta}_{1} C_{l} x_{5}\right)^{T} P C_{l} x_{5} \\
0 & =\left(c\left(\bar{\theta}_{2}\right) \bar{\theta}_{2} C_{l} y-\bar{\theta}_{1} C_{l} x_{5}\right)^{T} P c\left(\bar{\theta}_{2}\right) C_{l} y
\end{aligned}
$$

If the initial conditions are such that $y \not \equiv 0, x_{5} \not \equiv 0$, then $z=\phi(x)=\left[\tilde{x}, \bar{\theta}_{1}, \bar{\theta}_{2}\right]^{T}=0$ become an isolated root. Then for this particular solution, and noticing that 
$\bar{\theta}_{1}=\frac{\theta_{1}-\hat{\theta}_{1}}{\alpha}=0$ and $\bar{\theta}_{2}=\frac{\theta_{2}-\hat{\theta}_{2}}{\alpha}=0$, e.i. $\hat{\theta}_{1}=\theta_{1}$ and $\hat{\theta}_{2}=\theta_{2}$ the slow model writes as:

$$
\dot{x}=f(x)-U(x, 0) x=f(x),
$$

which is nothing else than the tuned system whose solutions $x(t)=x^{*}(t)$ are sinusoidal.

\subsection{Boundary layer fast subsystem}

For the evaluation of the boundary layer system in the finite time interval $t \in\left[t_{0}, t_{1}\right]$ Albea et al. [2007], we consider a particular solution of $y$ and $x_{5}$ expressed in the stretched time coordinates $\tau=\left(t-t_{0}\right) / \varepsilon$ : $y^{*}=$ $y^{*}\left(\tau, \omega, \varepsilon, t_{0}\right)$ and $x_{5}^{*}=x_{5}^{*}\left(\tau, \omega, \varepsilon, t_{0}\right)$, the fast subsystem (32) evaluated along such particular solution is,

$$
\begin{aligned}
& \frac{d}{d \tau} \breve{\widetilde{x}}_{1}=-\breve{\widetilde{x}}_{1} \\
& \frac{d}{d \tau} \breve{\widetilde{x}}_{2}=-\breve{\widetilde{x}}_{2} \\
& \frac{d}{d \tau} \breve{\widetilde{x}}_{5}=-\breve{\widetilde{x}}_{5}+c\left(\breve{\bar{\theta}}_{2}\right) \breve{\bar{\theta}}_{2} C_{l} y^{*}-\breve{\bar{\theta}}_{1} C_{l} x_{5}^{*} \\
& \frac{d}{d \tau} \breve{\bar{\theta}}_{1}=\tilde{x}_{5} x_{5}^{*} \\
& \frac{d}{d \tau} \breve{\bar{\theta}}_{2}=-c\left(\breve{\bar{\theta}}_{2}\right) \tilde{x}_{5} y^{*}
\end{aligned}
$$

we can compactly rewrite them as:

$$
\frac{d}{d \tau} \breve{z}=J\left(y^{*}, x_{5}^{*}\right) \breve{z}=J(\tau, \omega, \varepsilon) \breve{z}
$$

with

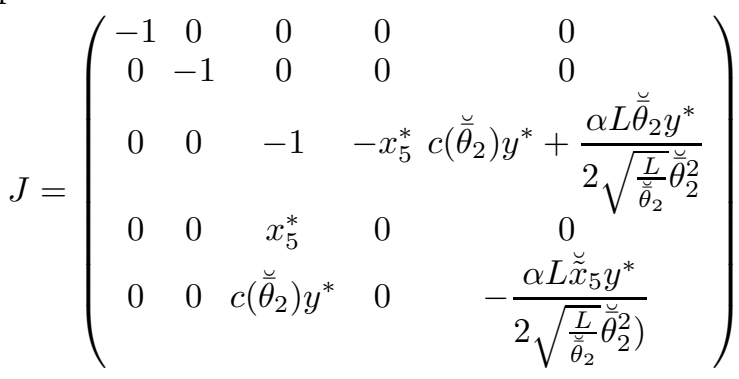

And the autonomous linear system

$$
\frac{d}{d \tau} \breve{z}=J(\tau, \omega, 0,) \breve{z}=J\left(y_{0}^{*}, x_{50}^{*}\right) \breve{z}
$$

Consider the $y_{0}^{*}, x_{50}^{*} \in D_{x}$, with $D_{x} \triangleq\left\{x:|y|=\mid x_{2}-\right.$ $\left.x_{4}\left|>\delta_{0}>0,\right| \check{x}_{5} \mid>\delta_{1}>0\right\}$, being $\delta_{0}$ and $\delta_{1}$ are constants. the above system has the following properties.

Property 2. The eigenvalues of $J\left(y^{*}, x_{5}^{*}\right)$, for $\left[t, \check{x}^{*}, z\right] \in$ $\left[t_{0}, t_{1}\right] \times D_{x} \times \mathbb{R}^{5}$, are all strictly negative.

By the Routh stability criterion is proved that the eigenvalues are strictly negative.

Tikhonov's theorem, see Khalil [2002], can now be advocated to summarize the previous result.

Theorem 1. There exists a positive constants $\varepsilon^{*}$ such that for all $y_{0}^{*} \in D_{x}$, and $0<\varepsilon<\varepsilon^{*}$, the singular perturbation problem of (31)-(32) has a unique solution $x(t, \varepsilon), z(t, \varepsilon)$ on $\left[t_{0}, t_{1}\right]$, and

$$
\begin{aligned}
x(t, \varepsilon)-x^{*}(t) & =O(\varepsilon) \\
z(t, \varepsilon)-\hat{z}^{*}(t / \varepsilon) & =O(\varepsilon)
\end{aligned}
$$

hold uniformly for $t \in\left[t_{0}, t_{1}\right]$, where $\hat{z}^{*}(\tau)$ is the solution of the boundary layer model (35). Moreover, given any $t_{b}>t_{0}$, there is $\varepsilon^{* *} \leq \varepsilon^{*}$ such that $z(t, \varepsilon)=O(\varepsilon)$ holds uniformly for $t \in\left[t_{\theta_{1}}, t_{1}\right]$ whenever, $\varepsilon<\varepsilon^{* *}$.

Extension of this result to infinite time interval, requires prove that the boundary layer system is exponential stable in a neighborhood of the tuned slow solution $x^{*}(t)$ for all $t \geq t_{0}$. This may not be a trivial demonstration, and it will be left for further investigation. Instead, we showed below using simulation the effectiveness of this approach.

An intuitive yet not completely rigorous explanation for the good resultant behavior can be given with the help of Fig. 3. Notice that the Hurwitz character of Jacobian (5.5) is only lost when $y=0$ and $x_{5}=0$ at the same instants. Since the fast motion, $z$, evolves with almost constant $y$ and $x_{5}$ (see Fig. 3), $y$ and $x_{5}$ will not reach the value zero during this motion provided that the initial condition is such that $y$ and $x_{5}$ are far enough from zero. Once the slow manifold is reached, the slow variable will evolve in the domain $z=0$. This domain corresponds to the case when the adaptation mechanism has reached its objective and parameter $\theta_{1}$ and $\theta_{2}$ are correctly estimated. In this domain $y$ and $x_{5}$ may reach the value zero but, intuitively, we can think that the system, once the adaptation law has reached the correct value, will present a behavior that is similar to the case of known load. For this last case stability is proved in Albea et al. [2006].

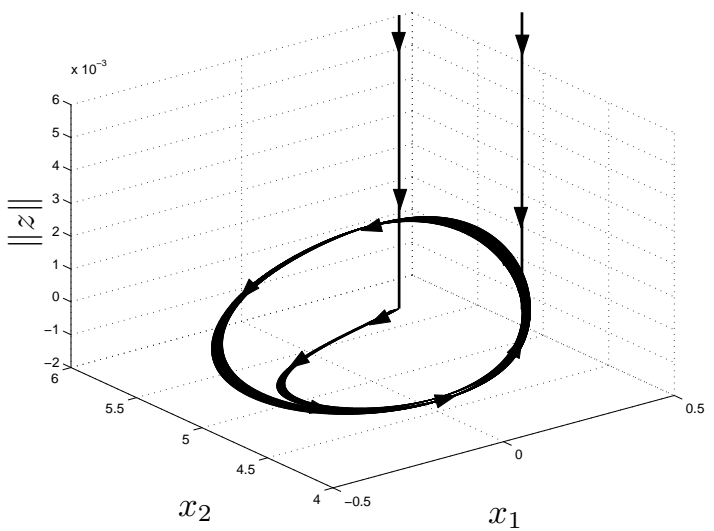

Fig. 3. Evolution of $\left(x_{1}, x_{2},\|z\|\right)$. The last part of the trajectory is in the plane $\|z\|=0$

\section{SIMULATIONS}

The following simulations are made considering $\mathrm{Vin}=$ $20 \mathrm{~V}, R_{0}=100 \Omega, L_{1}=L_{2}=1.5 \mathrm{mH}, C_{1}=C_{2}=100 \mu \mathrm{F}$. The desired output of the circuit is $V_{\text {out }}=40 \sin 50 t \mathrm{~V}$.

In order to obtain this voltage, the parameters are $\theta_{1}=$ $0.122, \theta_{2}=66.66, \varepsilon=10^{-8}, \omega=0.122, A=1, k=1.2$ and $\eta_{20}=\eta_{40}=0$. The ellipse parameters result according to Albea and Gordillo [2007] are $\eta_{10}=\eta_{30}=12.875, \mu=$ 0.372 . The load parameters are perturbed in two times, corresponding to the transitory and stationary states. In the instant $t=0 \mathrm{~s}$, the estimated value of parameter $\theta_{1}$ and $\theta_{2}$ will be $\hat{\theta}_{1}=0.135$ and $\hat{\theta}_{2}=71.43\left(R_{0}=110 \Omega\right.$ and $L_{R 0}=14 \mathrm{mH}$ ), i.e., a $15.35 \%$ and $10 \%$ error, respectively. Later, in the instant $t=6.12 \mathrm{~s}$ the parameters take the real value of $R_{0}=110 \Omega$ and $L_{R_{0}}=14 \mathrm{mH}$. 
Fig. 4 shows the output voltage evolution at the instant of the perturbation. Note that the output does not suffer any perturbation. The adaptation of the parameters $\theta_{1}$ and $\theta_{2}$ are represented in the Fig. 5 and Fig. 6, respectively.

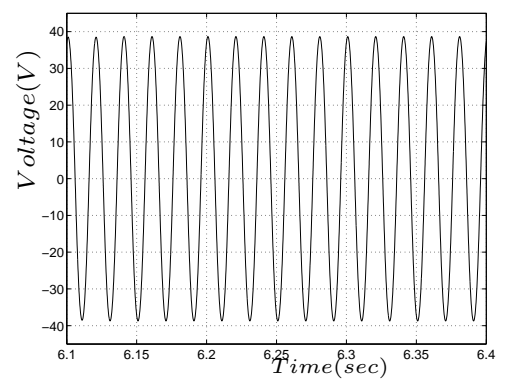

Fig. 4. Output voltage with adaptation of a perturbation of a $15.35 \%$ for $\theta_{1}$ and $10 \%$ for $\theta_{2}$ at the instant of the perturbation.
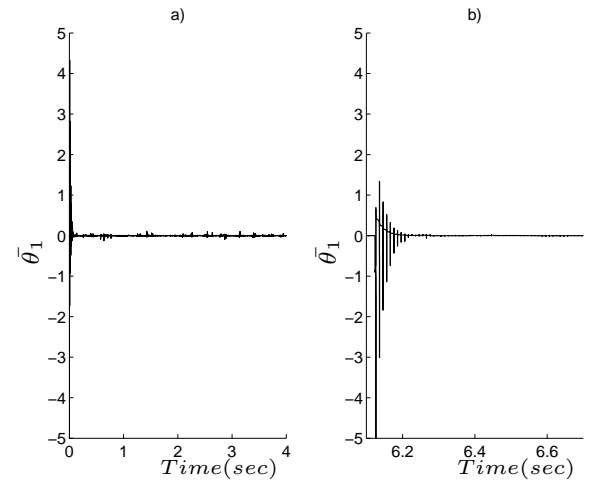

Fig. 5. Time-evolution of the fast variable $\overline{\theta_{1}}-\bar{\theta}_{1}{ }^{*}$
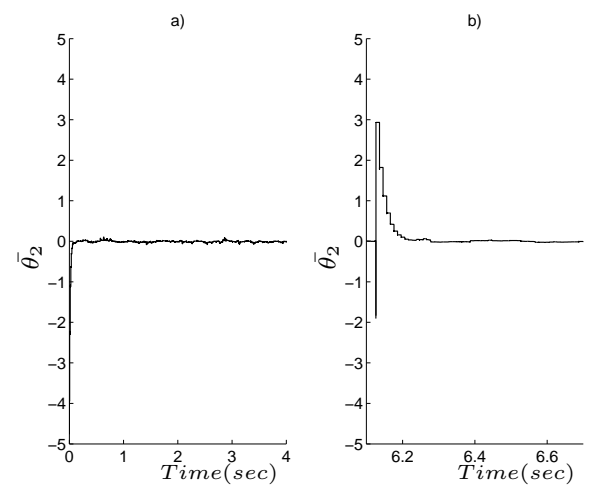

Fig. 6. Time-evolution of the fast variable $\overline{\theta_{2}}-{\overline{\theta_{2}}}^{*}$

\section{CONCLUSIONS}

An adaptive control for an unknown RL load is presented for a nonlinear boost inverter. The method is based on using a state observer and a quadratic Lyapunov function to one side of the inverter and by knowing that the state variables are measured. The stability of the complete system is proved putting the system in the standard singular perturbation form, hence we obtained a relationship between the adaptation gains, $\gamma$, the observer matrix parameter, $\alpha$, and the perturbed variable parameters, $\varepsilon$. Another important relationship between the perturbed variable parameter, $\varepsilon$, and the system frequency, $\omega$, was achieved in the analysis of the boundary layer fast subsystem. Finally, the stability is established by means of Tikhonov's theorem.

Open problem is the extension of this result to infinite time interval.

\section{REFERENCES}

C. Albea and F. Gordillo. Control of the boost DC-AC converter with RL load by energy shaping. Decision and Control, 2007 46th IEEE Conference on, pages 24172422, 2007.

C. Albea, C. Canudas-de Wit, and F. Gordillo. Adaptive Control of the Boost DC-AC Converter. pages 611-616, 2007.

Carolina Albea, Francisco Gordillo, and Javier Aracil. Control of the boost DC-AC converter by energy shaping. IEEE Industrial Electronics, IECON 2006-32nd Annual Conference on, pages 754-759, 2006.

RO Caceres and I. Barbi. A boost DC-AC converter: analysis, design, and experimentation. Power Electronics, IEEE Transactions on, 14(1):134-141, 1999.

H. El Fadil, F. Giri, M. Haloua, H. Ouadi, I. LAP, and F. Caen. Nonlinear and adaptive control of buck power converters. Decision and Control, 2003. Proceedings. 42nd IEEE Conference on, 5, 2003.

Hassan K. Khalil. Nonlinear Systems. Prentice Hall, third edition edition, 2002.

D. J. Pagano, J. Aracil, and F. Gordillo. Autonomous oscillation generation in the boost converter. In Proceedings of the $16^{\text {th }}$ IFAC World Congress, 2005.

P. Sanchis, A. Ursaea, E. Gubia, and L. Marroyo. Boost DC-AC Inverter: A New Control Strategy. Power Electronics, IEEE Transactions on, 20(2):343-353, 2005.

S.C. Tan, YM Lai, and CK Tse. Adaptive control schemes for stabilizing switching frequency of sliding mode controlled power converters. Power Electronics and Applications, 2005 European Conference on, page 8, 2005.

N. Vazquez, D. Cortes, C. Hernandez, J. Alvarez, J. Arau, and J. Alvarez. A new nonlinear control strategy for the boost inverter. Power Electronics Specialist Conference, 2003. PESC'03. 2003 IEEE 34th Annual, 3, 2003.

\section{Appendix A. OPERATION MODES}

The equations of two operation modes of the boost inverter system are

$$
\begin{gathered}
L \frac{d i_{L_{1}}}{d t}=-u v_{1}+V_{i n} \\
C \frac{d v_{C}}{d t}=u i_{L_{1}}-i_{R} \\
L_{R} \frac{d i_{L_{R}}}{d t}=v_{1}-v_{2}-i_{R} R
\end{gathered}
$$

Appendix B. CONTROL LAW

$$
\begin{aligned}
k_{1}\left(x, \theta_{1}, \theta_{2}\right)= & \frac{2\left(1+b^{2} x_{5}^{2}-b c \theta_{2} x_{2}\left(x_{2}-x_{4}\right)+b \theta_{1} \theta_{2} x_{5} x_{2}-b a \dot{\theta}_{2} x_{5} x_{2}\right)}{2\left(x_{2}+b x_{1} x_{5}\right)} \\
& +\frac{-c \dot{\theta}_{2} x_{2} x_{5}+2 k \Gamma\left(\eta_{2}-\eta_{20}\right)+2 \omega^{2}\left(\eta_{1}-\eta_{10}\right)}{2\left(x_{2}+b x_{1} x_{5}\right)} \\
k_{2}\left(x, \theta_{1}, \theta_{2}\right)= & \frac{2\left(1+b^{2} x_{5}^{2}+b c \theta_{2} x_{4}\left(x_{2}-x_{4}\right)-b \theta_{1} \theta_{2} x_{5} x_{4}+b a \dot{\theta}_{2} x_{5} x_{4}\right)}{2\left(x_{4}-b x_{3} x_{5}\right)} \\
& +\frac{c \dot{\theta}_{2} x_{4} x_{5}+2 k \Gamma\left(\eta_{4}-\eta_{40}\right)+2 \omega^{2}\left(\eta_{3}-\eta_{30}\right)}{2\left(x_{4}-b x_{3} x_{5}\right)}
\end{aligned}
$$

